\title{
2.2.3 Evangelikalismus in Lateinamerika ${ }^{1}$
}

Jens Köhrsen

\section{EinLEITUNG}

Evangelikale Protestanten bilden heute die zweitgrößte religiöse Gruppe in Lateinamerika. Damit sind sie zu einer zentralen Größe im religiösen Feld Lateinamerikas und zum bedeutsamsten Konkurrenten des einstigen religiösen Monopolisten, der katholischen Kirche, geworden. Der vorliegende Beitrag skizziert Geschichte, Verbreitung und unterschiedliche Formen des evangelikalen Protestantismus in Lateinamerika. Er beginnt mit einer historischen Darstellung der Ausbreitung des Protestantismus in Lateinamerika, die in drei Wellen erfolgt. Basierend auf den drei Wellen wird eine Unterteilung in liberalen und evangelikalen Protestantismus vorgenommen. Hierbei wird der evangelikale Protestantismus vom liberalen Protestantismus durch einen Missionsfokus, restriktivere Moralvorstellungen sowie die Betonung von Konversion als Neuanfang unterschieden. Der evangelikale Protestantismus kann wiederum in nicht-charismatische und charismatische (pfingstliche) Strömungen unterteilt werden. Da der charismatische Evangelikalismus heute die dominanteste Form des Evangelikalismus in Lateinamerika ist, liegt der Fokus des Beitrags besonders auf dieser Strömung. So werden die Ausbreitungsgeschichte der Pfingstbewegung und verschiedene theoretische Modelle vorgestellt, die deren enormen Erfolg in den Unterschichten Lateinamerikas erklären. Der Beitrag endet mit einem Ausblick auf die mögliche Weiterentwicklung der Pfingstbewegung.

\section{Typen und Verbreitung des Protestantismus in Lateinamerika}

Die ersten Protestanten erreichen die Spanischen und Portugiesischen Kolonien in Lateinamerika bereits im 16. Jahrhundert, werden jedoch in den meisten

1 | Für ihre hilfreichen Kommentare zu diesem Beitrag bin ich Frederik Elwert, Martin Radermacher, Jens Schlamelcher und Rahel Weber sehr dankbar. 
Fällen beim Versuch, sich dauerhaft in den Herrschaftsgebieten der spanischen und portugiesischen Krone niederzulassen, durch militärische Kampagnen der kolonialen Mächte und die Inquisition exterminiert (Prien 2012, S. 144-147, 199-210). Im Zuge von kommerziellen Handelsabkommen zwischen der spanischen Krone und protestantischen Ländern im 17. Jahrhundert lockert sich die Position gegenüber Protestanten, die sich zunehmend in den Kolonien Amerikas niederlassen können. Eine verstärkte Öffnung vollzieht sich schließlich im 19. Jahrhundert unter den liberalen Regierungen der nun unabhängigen amerikanischen Staaten. Da sie sich vom Protestantismus einen positiven Einfluss auf die zivilisatorische und ökonomische Entwicklung Lateinamerikas erhoffen, werden gezielte Versuche unternommen, die Einwanderung von Protestanten zu fördern (Prien 2012, S. 356ff.). Besonders in Südamerika siedeln sich Anglikaner, Lutheraner, Presbyterianer, Reformierte etc. aus dem deutschsprachigen Raum, Holland, Dänemark und England an. Jedoch gehen kaum Missionierungsanstrengungen von diesen protestantischen Gruppen aus. Die zweite Welle des Protestantismus in Lateinamerika beginnt ab Mitte des 19. Jahrhunderts mit dem missionarischen Protestantismus (Prien 2012, S. 375ff.). Zahlreiche Gruppierungen aus Nordamerika und Nordeuropa (z.B. Baptisten), welche heute mehrheitlich den evangelikalen Strömungen des Protestantismus zugerechnet werden, drängen in >Faith Missions « mit dem Ziel der Bekehrung möglichst großer Bevölkerungsteile nach Lateinamerika. Jedoch ist auch ihr Erfolg vorerst begrenzt. Mit dem beginnenden 20. Jahrhundert breitet sich in einer dritten Welle des Protestantismus in Lateinamerika die Pfingstbewegung aus, die ebenfalls den evangelikalen Strömungen des Protestantismus zugerechnet wird (Anderson 2004, S. 64ff.; Wynarczyk 1999). Ab der zweiten Hälfte des 20. Jahrhunderts erfährt die Pfingstbewegung in vielen Regionen Lateinamerikas ein starkes Mitgliederwachstum und trägt so wesentlich zur Ausbreitung des Protestantismus in Lateinamerika bei (siehe weiter unten für nähere Informationen zur Ausbreitungsgeschichte der Pfingstbewegung).

Gemäß einer in den Jahren 2013/2014 durchgeführten Studie des Pew Research Centers (Pew Research Center 2014) ist fast jeder fünfte Lateinamerikaner heute Protestant: 19 Prozent der Befragten bezeichneten sich als Protestanten. Mehr als die Hälfte dieser Befragten sind aus dem Katholizismus stammende Konvertiten. Diese Zahlen verdeutlichen, dass es sich beim Protestantismus heute um einen ernstzunehmenden Marktrivalen des einstigen >Monopolisten< katholische Kirche handelt (Chesnut 2003). Bei der Betrachtung einzelner Länder fallen jedoch starke Schwankungen auf: Während beispielsweise 41 Prozent der Bevölkerung in Guatemala und Honduras Protestanten sind, sind es nur sieben Prozent in Paraguay und neun Prozent in Mexiko (Pew Research Center 2014, S. 14). 
Der Protestantismus in Lateinamerika kann schematisch in verschiedene Strömungen unterteilt werden. ${ }^{2}$ Die vorgeschlagene Unterteilung folgt dabei den unterschiedlichen historischen Wellen des Protestantismus. Hierbei können zunächst die evangelikalen Strömungen vom liberalen - auch oft als historischer oder >mainstream < bezeichneten - Protestantismus (z.B. Lutheraner, Reformierte, Presbyterianer, Anglikaner) abgegrenzt werden, der größtenteils mit der ersten Welle nach Lateinamerika gelangt: Die evangelikalen Strömungen unterscheiden sich vom liberalen Protestantismus durch ihr restriktiveres Moralverständnis (z.B. Sexualmoral) sowie ihre missionarische Ausrichtung und den Fokus auf Konversion als Neubeginn (Stoll 1990, S. 4). ${ }^{3}$ Der evangelikale Protestantismus lässt sich wiederum in zwei große Strömungen unterteilen: charismatische und nicht-charismatische Formen des Evangelikalismus. Der charismatische Evangelikalismus unterscheidet sich vom nicht-charismatischen Evangelikalismus besonders durch den Glauben an das Wirken des Heiligen Geistes im Leben der Gläubigen, wie weiter unten ausgeführt wird. Diese Strömung tritt in Lateinamerika vornehmlich in Form der Pfingstbewegung auf. Insgesamt lassen sich also drei Strömungen des Protestantismus unterscheiden:

- Historischer Protestantismus

- Nicht-charismatische Formen des Evangelikalismus

- Charismatische Formen des Evangelikalismus (Pfingstbewegung)

Eine Schwierigkeit bei der empirischen Arbeit mit der vorgeschlagenen Unterteilung ergibt sich aus dem Umstand, dass die Begriffe >Evangélicos < und >Protestantes in Lateinamerika als Sammelbegriffe für alle Protestanten verwendet werden, ohne hierbei Unterschiede zwischen Pfingstlern (charismatischen Evangelikalen), nicht-charismatischen Evangelikalen und historischen/liberalen Protestanten zu machen. >Evangélicos < und >Protestantes < werden somit als austauschbare Begriffe verwendet, die sich für die Befragten auf den gleichen Gegenstand beziehen (vgl. Pew Research Center 2014, S. 7). Das Auseinanderdriften der wissenschaftlichen und alltäglichen Definition erschwert dabei die Zuweisung von Denominationen, Kirchen und Gläubigen zu den einzelnen Strömungen.

2 | Solch eine Unterteilung geht immer mit Unsauberkeiten einher, so dass sich einzelne Kirchen und Denominationen häufig nicht klar einer bestimmten Strömung zuweisen lassen.

3 | Weiterhin werden innen oft konservative politische Tendenzen zugeschrieben. Diese politische Zuordnung gilt jedoch als umstritten (siehe zum Beispiel Stoll 1990, S. 19ff.; Stewart-Gambino/Wilson 1997). 


\section{Nicht-Charismatische Formen des Evangelikalismus}

Bei den nicht-charismatischen Formen des Evangelikalismus handelt es sich vornehmlich um jene missionarisch orientierten Denominationen und Gruppierungen, die sich besonders mit und ab der zweiten Welle in Lateinamerika ansiedeln. Beispiele für nicht-charismatische evangelikale Denominationen sind die Baptisten und die Plymouth-Brüderbewegung (Hermanos Libres). Aufgrund der starken Ausbreitung der Pfingstbewegung dürften heute weit weniger als ein Drittel der Protestanten nicht-charismatische Evangelikale sein (Pew Research Center 2014). ${ }^{4}$ Bedingt durch ihre schnelle und massive Verbreitung hat sich die wissenschaftliche Arbeit der letzten Jahrzehnte fast ausschließlich auf die Pfingstbewegung konzentriert (Chesnut 1997; Freston 1998; Freston 1999; Martin 1995; Martin 1990; Martin 2002; Smilde 2007), während nicht-charismatische evangelikale Strömungen weitgehend unberücksichtigt geblieben sind. So liegen heute nur verhältnismäßig wenige Studien zu den nicht-charismatischen Evangelikalen in Lateinamerika vor (Wynarczyk 2007). Die starke Präsenz der Pfingstbewegung und ihre Anziehungskraft haben die nicht-charismatischen Formen des Evangelikalismus nicht unberührt gelassen. So haben sich auch in vielen der ursprünglich nicht-charismatischen Kirchen, die der Pfingstbewegung häufig äußerst kritisch gegenüberstanden, charismatische Erneuerungsbewegungen vollzogen (Anderson 2004). Vielfach gingen diese Erneuerungsbewegungen in den Gemeinden mit Konflikten zwischen pro-charismatischen und anti-charismatischen Lagern einher, die in nicht wenigen Fällen zu Spaltungen innerhalb der Gemeinden geführt haben. Aus diesen Spaltungen heraus haben sich neue, charismatisch ausgerichtete Gemeinden gebildet, die sich selbst nach wie vor als Baptisten oder PlymouthBrüderschaften bezeichnen. Ebenso haben viele der bestehenden Gemeinden eine charismatische Wende vollzogen, die sie heute in die Nähe der Pfingstbewegung rückt. ${ }^{5}$

4 I In diese Rechnung sind noch die historischen Protestanten einzubeziehen, so dass der Anteil der nicht-charismatischen Evangelikalen wahrscheinlich weit unter einem Drittel der Protestanten liegen wird.

5 | Hier stellt sich die Frage, ob diese Gruppierungen als Teil der Pfingstbewegung zu betrachten sind. Je nach Definition der Pfingstbewegung wird man diese Frage bejahen oder eine Unterscheidung zwischen Pfingstbewegung und anderen Formen des charismatischen Protestantismus einführen. 


\section{Charismatische Formen des Evangelikalismus: Die Pfingstbewegung}

\section{Merkmale der Pfingstbewegung}

Die Pfingstbewegung ist eine evangelikale Erneuerungsbewegung, die am Anfang des 20. Jahrhunderts entsteht und deren Mitgliederzahl in Lateinamerika zu Anfang des 21. Jahrhunderts auf 141 Millionen geschätzt wird (Barrett/Johnson/Guidry/Crossing 2001, S. 14, 20). Da es innerhalb der Pfingstbewegung keine allumfassende Dachorganisation gibt, zerfällt sie in viele kleine und manchmal größere Gemeinden und Gemeindeverbände, die voneinander unabhängig existieren und unterschiedliche pfingstliche Praxisstile hervorbringen (Robbins 2004, S. 125; Semán 2000, S. 160; Willaime 1999, S. 14.). Trotz der Vielzahl der Manifestierungsformen, die sich hinter dem Begriff >Pfingstbewegung< verbergen (Anderson 2004, S. 10, 13; Robbins 2004, S. 119; Schäfer 2009b, S. 555; Willaime 1999, S. 5), werden für gewöhnlich bestimmte, einende Eigenschaften mit diesem verbunden: Primär zeichnet sich die Pfingstbewegung durch den Glauben an das Wirken des Heiligen Geistes aus. So glauben Pfingstler, dass sich der Heilige Geist in ihrem diesseitigen Leben manifestiert und ihnen bestimmte Gaben verleihen kann, wie etwa das Sprechen in fremden Sprachen (Zungenrede), Heilung von Krankheiten, Geisteraustreibung und Prophetie. Der Glaube an diese Gaben kann sich im Gottesdienst in Form von Zungenrede und anderen Praktiken, die sich auf den Heiligen Geist beziehen (z.B. Exorzismen), äußern (Anderson 2004, S. 14; Chesnut 1997, S. 6, 47; Corten 1995, S. 10-12, 153-154; Cox 2001, S. 81-110; Freston 1998, S. 340; Jenkins 2007, S. 8, 73; Lehmann 2003, S. 479; Parker 1996, S. 142; Schäfer 1992, S. 5357; Schäfer 1998, S. 67; Schäfer/Tovar 2009, S. 5-6). Weiterhin sind die pfingstlichen Gottesdienste für ihre Ausgelassenheit, Feierlichkeit und Emotionalität bekannt. Oft ähneln sie einer emotionalen Achterbahnfahrt mit Momenten der Besinnlichkeit, Ruhe und Trauer, die abgelöst werden von Momenten der Freude, Hoffnung und Ausgelassenheit. Musikalisch werden die verschiedenen Momente häufig von christlichem Rock, eindringlichen Pop-Balladen sowie lateinamerikanischen Rhythmen (wie etwa Cumbia) begleitet (Robbins 2004, S. 125-126; Schäfer 1988, S. 71; Wynarczyk/Semán/Majo 1995, S. 7-8; siehe Emling/Schira, Kapitel 3.3.8 »Evangelikalismus und populärkulturelle Musik«). Ein weiteres gemeinsames Moment ist der starke Fokus auf Missionierung. So wird von Anhängern der Bewegung erwartet, dass sie in ihrem sozialen Umfeld aktiv für die Konversion zum Protestantismus werben (Robbins 2004, S. 124; Willaime 1999, S. 6). Dabei wird die Konversion als ein radikaler Neubeginn verstanden, der mit dem bisherigen Leben bricht (Chesnut 1997, S. 73; Lehmann 2003, S. 479: Robbins 2004, S. 127-129; Schäfer 2009b, S. 565). 


\section{Geschichte und Ausbreitung in Lateinamerika}

Die Pfingstbewegung gelangt über US-amerikanische und europäische Missionare im frühen 20. Jahrhundert nach Lateinamerika. Trotz ihrer frühen Ankunft bleibt die Pfingstbewegung in den meisten Ländern Lateinamerikas zunächst eine eher kleine und randständige Bewegung. Erst ab den 1950er Jahren vollzieht sich eine Massenausbreitung. Je nach Land variiert der Zeitpunkt der Expansion: Während eine verstärkte Ausbreitung der Pfingstbewegung bereits ab 1940 in Chile einsetzt, beginnt sie im Nachbarland Argentinien erst nach dem Ende der letzten Diktatur ab Mitte der $1980 e r$ Jahre (Bastian 1994, S. 115; Chesnut 1997, S. 39; Cleary/Steigenga 2004, S. 12; 1999, S. 147; Gill 1999, S. 287288; Hurtado Cruchaga 1941, S. 104-127; Jenkins 2007, S. 73; Martin 1990, S. 49; Martin 2002, S. 71; Wynarczyk/Semán/Majo 1995, S. 7ff., Saracco 1989, S. 299). Dabei wird die massive Ausbreitung von Innovationen innerhalb der Pfingstbewegung begleitet: Viele Kirchen beginnen auf moderne Massenmedien (Radio, Fernsehen und später Internet) für die Verbreitung des Evangeliums zurückzugreifen und orientieren sich zunehmend an dem sogenannten Wohlstandsevangelium (>Prosperity-Gospel//teología de la prosperidad<) und der spirituellen Kriegsführung (’spiritual warfare</>guerra espiritual<) als Reinigung von bösartigen, spirituellen Kräften. Kirchen, die sich diesen Innovationen verschreiben, werden häufig als >neo-pfingstlich Verwendung des Begriffs >neo-pfingstlich (Freston 1999, S. 150-152; Jaimes 2007; Mansilla 2008; Willaime 1999, S. 8-9), so dass die unterschiedlichen Ausprägungen häufig unter dem Sammelbegriff >Pfingstbewegung< erfasst werden, ohne eine Trennung zwischen >klassischen< und `neo-pfingstlichen< Strömungen vorzunehmen (Koehrsen 2016).

Heute sind laut der Studie des Pew-Research Centers knapp zwei Drittel der lateinamerikanischen Protestanten Pfingstler. Auch hier unterscheiden sich die Länder: Der pfingstliche Anteil der Protestanten variiert zwischen knapp 50 Prozent (in Bolivien, Peru und Uruguay) und über 80 Prozent (in der Dominikanischen Republik, Brasilien, Panama) (Pew Research Center 2014, S. 62). Die Zahlen illustrieren den Erfolg der Pfingstbewegung, die heute nicht nur den Großteil der Protestanten ausmacht, sondern auch das erfolgreichste religiöse Angebot mit Blick auf Neuzugänge ist. Durch ihren beträchtlichen Erfolg bei der Gewinnung neuer Mitglieder ist es vor allem diese Form des Protestantismus, die heute den zentralen Konkurrenten des Katholizismus bildet. Die Pfingstbewegung hat sich jedoch nicht in allen Teilen der Gesellschaft gleichermaßen durchgesetzt. So hat sich die Pfingstbewegung besonders in den Unterschichten Lateinamerikas verbreitet, während ihre Ausbreitung in den Mittelschichten in vielen Ländern (z.B. Argentinien) bisher wesentlich zögerlicher verlaufen ist (Anderson 2004, S. 59, 282; Bastian 1997, S. 59-72, 61-68; Chesnut 1997, S. 17; Chesnut 2003, S. 39-43; Freston 1998, S. 338, 341-342; Köhr- 
sen 2014; Lehmann 1996, S. 210-214; Mariz 1994, S. 35; Martin 1990, S. 53, 202; Martin 2002, S. 1, 20, 78; Schäfer 2009a, S. 48; Schäfer 2010, S. 93, 98-99).

\section{Gründe für die Ausbreitung der Pfingstbewegung in der Unterschicht}

Verschiedene Theorien versuchen, die erfolgreiche Ausbreitung der Pfingstbewegung in den Unterschichten Lateinamerikas zu erklären. Dabei überwiegen hauptsächlich zwei Erklärungsstränge: die Deprivationstheorie und der Marktansatz.

Laut der Deprivationstheorie zieht die Pfingstbewegung besonders jene Menschen an, die von Armut und damit einhergehenden Problemen (z.B. Krankheit und mangelnde medizinische Versorgung) betroffen sind, da sie ihnen Strategien des Umgangs mit ihren armutsbezogenen Schwierigkeiten anheimstellt. Einerseits bietet die Pfingstbewegung ihren Anhängern Hoffnung auf spirituelle Heilung und Besserung durch den Glauben an den Heiligen Geist, andererseits rüstet sie die Gläubigen mit einem moralischen Leitfaden aus, der ihnen bei der Restrukturierung des eigenen Lebens (z.B. Verzicht auf Alkohol und Drogen) behilflich ist (Chesnut 1997; Mariz 1994). Gerade der Bruch mit der Vergangenheit und der erlebte Neubeginn des Lebens, das sich von nun an auf den Glauben und eine strikte moralische Lebensführung stützt, helfe, so die Ansätze, bei dem Umgang mit Verhältnissen totaler Armut (siehe für einen Überblick Koehrsen 2015).

Marktansätze hingegen betonen, dass die katholische Kirche als zentraler religiöser Anbieter sich über lange Zeit hinweg auf die nationalen Oberschichten konzentriert und so die religiöse Nachfrage der Unterschichten ausgeblendet habe (Gill 1994; Chesnut 2003; Smilde 2005). Zugleich verfügt die Pfingstbewegung über eine breite Palette an unterschiedlichen religiösen Produkten, um unterschiedliche Präferenzen anzusprechen, wie Chesnut betont:

"In effect, there are so many varieties of Pentecostalism available in the religious marketplace that consumers can choose their brands according to preferences in gender, class, age, musical tastes, and so on " (Chesnut 2003, S. 61).

Neben diesen Faktoren spielt auch das grundsätzliche Passungsverhältnis zwischen den religiösen Präferenzen der Unterschicht und dem $/ \mathrm{n}$ angebotenen Produkt(en) eine Rolle. So stellt die Pfingstbewegung eine attraktive Rekombination verschiedener Elemente der populären Religiosität dar. Populäre Religiosität (>religiosidad popular<) bezieht sich im Lateinamerikanischen Kontext auf jene Religionsformen, die besonders in den Unterschichten Lateinamerikas verbreitet sind. Die Gemeinsamkeiten, die diese Religionsformen aufweisen, zeugen von einer eigenen Logik der Religiosität in den Unterschichten: z.B. Verschränkung 
von Transzendenz und Immanenz, Festlichkeit der religiösen Praxis, Fokus auf Verbesserungen im Diesseits (Ameigeiras 2008; Parker 1996). Durch eine attraktive Rekombination dieser religiösen Elemente und Tendenzen- gelingt es der Pfingstbewegung, erfolgreich Menschen aus der Unterschicht anzusprechen (Koehrsen 2016; Robbins 2004; Semán 2000; Semán 2004).

\section{Die künftige Evolution der Pfingstbewegung}

Neben dem beträchtlichen Erfolg in der Unterschicht gelingt es der Pfingstbewegung mittlerweile, sich zunehmend auch in den Mittelschichten Lateinamerikas auszubreiten. Oft sind es moralisch weniger restriktive Kirchen, aus denen spirituelle Heilungspraktiken und Zungenrede zunehmend verbannt werden, die die Mittelschichten anziehen (Corten 1995; Freston 1997; Gooren 2011; Martin 1995; Martin 2006). Somit stellt sich die Frage, ob sich im Zuge der Ausbreitung der Pfingstbewegung in der Mittelschicht sowie des sozialen Aufstiegs von Pfingstlern der zweiten und dritten Generation, langfristig eine neue - möglicherweise säkularere und gesellschaftlich salonfähigere - Form des Pfingstlertums in Lateinamerika ausbreiten wird (Köhrsen 2014; Koehrsen 2016).

\section{Weiterführende Literatur}

Chesnut, Andrew R. (1997): Born Again in Brazil. The Pentecostal Boom and the Pathogens of Poverty, New Brunswick, NJ.

Chesnut präsentiert eine spannende ethnographische Untersuchung über die Pfingstbewegung in Brasilien, die auf anschauliche Weise vermittelt, warum und wie Menschen aus der Lateinamerikanischen Unterschicht zur Pfingstbewegung konvertieren. Dabei vertritt der Autor die These, dass Konversionen zur Pfingstbewegung durch armutsbezogene Probleme bedingt seien.

Freston, Paul (1998): Pentecostalism in Latin America: Characteristics and Controversies, in: Social Compass 45, S. 335-358.

Der Aufsatz beschreibt die Pfingstbewegung in Lateinamerika. Hierbei behandelt der Autor unter anderem die unterschiedlichen Wellen der Pfingstbewegung, die schwierige Unterscheidung zwischen Neo-Pfingstlern und klassischen Pfingstlern sowie unterschiedliche Theorien zur Ausbreitung der Pfingstbewegung.

Eine Untersuchung über die Generation nach dem >Babyboom Auswirkung auf die amerikanische Religion, sowie eine Prognose für die Wirkung dieser Generation auf die Zukunft der amerikanischen Religion. 
Martin, David (1990): Tongues of fire. The explosion of Protestantism in Latin America, Oxford.

Mit Blick auf die Erforschung der Expansion der Pfingstbewegung in Lateinamerika ist das Buch von Martin heute ein Klassiker. Der Autor vertritt die These, dass die Ausbreitung der Pfingstbewegung zu Modernisierungsprozessen in Lateinamerika beitrage.

\section{BibliogRAPHIE}

Ameigeiras, Aldo Rubén (2008): Religiosidad popular. Creencias religiosas populares en la sociedad argentina, Buenos Aires.

Anderson, Allan (2004): An Introduction to Pentecostalism. Global Charismatic Christianity, Cambridge.

Barrett, David B./Johnson, Todd M./Guidry, Christopher R./Crossing, Peter F (2001): World Christian trends, AD 30-AD 2200. Interpreting the annual Christian megacensus, Pasadena, Calif.

Bastian, Jean-Pierre (1994): La Mutación Del Protestantismo Latinoamericano. Una Perspectiva socio-histórica. in: Gutiérrez, Tomás (Hg.). Protestantismo Y Cultura en América Latina. Aportes Y Proyecciones, Quito, S. 115-133.

Bastian, Jean-Pierre (1997): La mutación religiosa de América Latina. Para una sociología del cambio social en la modernidad periférica, México.

Chesnut, Andrew R. (1997): Born Again in Brazil. The Pentecostal Boom and the Pathogens of Poverty, New Brunswick, NJ.

Chesnut, Andrew R. (2003): Competitive Spirits. Latin America's new religious economy, Oxford.

Cleary, Edward L./Steigenga, Timothy J. (2004): Resurgent voices: Indians, Politics, and Religion in Latin America. in: Cleary, Edward L./Steigenga, Timothy J. (Hg.). Resurgent Voices in Latin America. Indigenous Peoples, Political Mobilization, and Religious Change, New Brunswick, NJ, London, S. 1-24.

Corten, André (1995): Le pentecôtisme au Brésil. Émotion du pauvre et romantism théologie, Paris.

Cox, Harvey (2001): Fire from heaven. The rise of pentecostal spirituality and the reshaping of religion in the twenty-first century, Cambridge, Mass.

Freston, Paul (1997): Charismatic Evangelicals in Latin America: Mission and Politics on the Frontiers of Protestant Growth. in: Hunt, Stephen J./Hamilton, Malcolm/Walter, Tony (Hg.). Charismatic Christianity. Sociological perspectives, New York, S. 184-204.

Freston, Paul (1998): Pentecostalism in Latin America: Characteristics and Controversies, in: Social Compass 45, S. 335-358. 
Freston, Paul (1999): Neo-Pentecostalism in Brazil: Problems of definition and struggle for hegemony, in: Archives de Sciences Sociales des Religions 44, S. $145^{-161 .}$

Gill, Anthony (1999): Government Regulation, Social Anomie and Protestant Growth in Latin America: A Cross-National Analysis, in: Rationality and Society 11, S. 287-316.

Gill, Anthony J. (1994): Rendering unto Caesar? Religious competition and Catholic political strategy in Latin America, 1962-79, in: American Journal of Political Science, S. 403-425.

Gooren, Henri (2011): The Pentecostalization of Religion and Society in Latin America: First Findings from Chile, Milwaukee.

Hurtado Cruchaga, Alberto (1941): Es Chile un país católico?

Jaimes, Ramiro (2007): Neopentecostales in Tijuana. in: La Torre, Renée de/ Gutiérrez, Cristina (Hg.). Atlas de la diversidad religiosa en México, México, S. 305-311.

Jenkins, Philip (2007): The next Christendom. The rise of global Christianity, Oxford, New York.

Koehrsen, Jens (2015): Pentecostal Improvement Strategies. A Comparative Reading on African and South American Pentecostalism. in: Heuser, Andreas (Hg.). Pastures of plenty. Tracing religio-scapes of prosperity gospel in Africa and beyond, Frankfurt a.M., S. 49-64.

Koehrsen, Jens (2016): Middle Class Pentecostalism in Argentina. Inappropriate Spirits, Leiden/Boston.

Köhrsen, Jens (2014): Religiöse Geschmäcker und Stile als Distinktionsmittel in der argentinischen Pfingstbewegung. in: Löw, Martina (Hg.). Vielfalt und Zusammenhalt. Verhandlungen des 36. Kongresses der Deutschen Gesellschaft für Soziologie in Bochum und Dortmund 2012, Frankfurt a.M.

Lehmann, David (1996): Struggle for the spirit. Religious transformation and popular culture in Brazil and Latin America, Cambridge.

Lehmann, David (2003): Disendencia y Confermismo en los movimientos religiosos: ¿Qué diferencia hay entre la renovación carismática católica y las iglesias pentecostales?, in: Concilium - Revista internacional de Teología, S. 477-495.

Mansilla, Miguel Ángel (2008): Pluralismo, subjetivización y mundanización. El impacto de la secularización en el neopentecostalismo chileno, in: Polis: Revista de la Universidad Bolivariana 19.

Mariz, Cecília Loreto (1994): Coping with Poverty. Pentecostals and Christian Base Communities in Brazil, Philadelphia.

Martin, Bernice (1995): New Mutations of the Protestant Ethic among Latin American Pentecostals, in: Religion 25, S. 101-117.

Martin, Bernice (2006): The Aesthetics of Latin American Pentecostalism: The Sociology of Religion and the Problem of Taste. in: Arweck, Elisabeth/ 
Keenan, William J.F. (Hg.). Materialising religion. Expression, performance, and ritual/[edited by] Elisabeth Arweck \& William J.F. Keenan, Aldershot, Hants, England, S. 138-16o.

Martin, David (1990): Tongues of fire. The explosion of Protestantism in Latin America, Oxford.

Martin, David (2002): Pentecostalism. The world their parish, Oxford.

Parker, Cristián Gumucio (1996): Popular religion and modernization in Latin America. A different logic, Maryknoll, NY.

Pew Research Center (2014): Religion in Latin America. Widespread Change in a Historically Catholic Region. www.pewresearch.org, Stand: 20.08.2015.

Prien, Hans-Jürgen (2012): Christianity in Latin America: Revised and Expanded Edition.

Robbins, Joel (2004): The Globalization of Pentecostal and Charismatic Christianity, in: Annual Review of Anthropology 33, S. 117-143.

Saracco, José Norberto (1989): Argentine Pentecostalism its History and Theology, Birmingham.

Schäfer, Heinrich (1988): Religión dualista causada por antagonismos sociales. Trasfondos sociales del protestantismo en Centroamerica, in: Boletín de Estudios Latinoamericanos y del Caribe 45, S. 69-90.

Schäfer, Heinrich (1992): Protestantismo y crisis social en América Central, San José.

Schäfer, Heinrich (1998): ¡Oh Señor de los Cielos, danos poder en la tierra! El fundamentalismo y los carismas: La reconquista del campo de acción en América Latina, in: Revista Latinoamerica De Teologia 15, S. 61-78.

Schäfer, Heinrich (2009a): La generación del sentido religiosos. observaciones acerca de la diversidad pentecostal in América Latina, in: Chiquete, Daniel/ Orellano, Luis (Hg.). Voces del pentecostalismo latinoamericano III. Identidad, teologia e historia, Santiago de Chile, S. 45-72.

Schäfer, Heinrich (2009b): Pfingstbewegung: Sozialer Wandel und religiöser Habitus, in: Bertelsmann Stiftung (Hg.). Woran glaubt die Welt? Analysen und Kommentare zum Religionsmonitor 2008, Gütersloh, S. 553-608.

Schäfer, Heinrich (2010): Religion in der konfliktiven Moderne Lateinamerikas, in: Reder, Michael/Rugel, Matthias (Hg.). Religion und die umstrittene Moderne, Stuttgart, S. 89-113.

Schäfer, Heinrich/Tovar, Adrián (2009): Religiöser Glaube und gesellschaftliches Handeln in der lateinamerikanischen Pfingstbewegung: zu empirischen und theoretischen Aspekten des aktuellen Forschungsstandes, Bielefeld. www.uni-bielefeld.de/theologie/CIRRuS-downloads/Schaefer-Tovar _2009_Forschung-Pent-LA-kurz_CIRRuS_WP7.pdf, Stand: 29.12.2015.

Semán, Pablo (2000): »El pentecostalismo y la religiosidad de los sectores populares«, in: Svampa, Maristella (Hg.) Desde abajo: La transformación de las 
identidades sociales, Buenos Aires. in: Svampa, Maristella/Auyero, Javier/ Isla, Alejandro Raúl (Hg.). Desde abajo. La transformación de las identidades sociales, Buenos Aires, S. 155-180.

Semán, Pablo (2004): La religiosidad popular. Creencias Y Vida Cotidiana, Buenos Aires.

Smilde, David (2005): A qualitative comparative analysis of conversion to Venezuelan evangelicalism: How networks matter, in: American Journal Of Sociology 111, S. 757-796.

Smilde, David (2007): Reason to believe. Cultural agency in Latin American evangelicalism, Berkeley, Calif.

Stewart-Gambino, Hannah W./Wilson, Everett (1997): Latin American Pentecostals: Old Stereotypes and New Challenges, in: Cleary, Edward L./StewartGambino, Hannah W. (Hg.). Power, politics, and Pentecostals in Latin America, Boulder, Colo., S. 227-246.

Stoll, David (1990): Is Latin America turning Protestant? The Politics of Evangelical Growth, Berkeley, Calif.

Willaime, Jean-Paul (1999): Le Pentecôtisme. Contours et paradoxes d'un protestantisme émotionnel/Pentecostalism: Outlines and Paradoxes of an Emotional Form of Protestantism, in: Archives de Sciences Sociales des Religions 105 , S. 5-28.

Wynarczyk, Hilario (1999): La difusión de las Iglesias Protestantes, Evangélicas y Pentecostales en la Argentina y el Brasil. Constantes y rupturas, in: Boletín de Lecturas Sociales y Económicas (Universidad Católica Argentina) 6.

Wynarczyk, Hilario (2007): Partidos políticos evangélicos conservadores bíblicos en la Argentina: formación y ocaso 1991-2001, in: Civitas - Revista de Ciências Sociais 6, S. 11.

Wynarczyk, Hilario/Semán, Pablo/Majo, Mercedes de (1995): Panorama Actual del Campo Evangélico en Argentina: Un Estudio Sociológico, Buenos Aires. 